\title{
Enhancement of human skin facial revitalization by moringa leaf extract cream
}

\author{
Atif Ali $^{1,2}$, Naveed Akhtar ${ }^{2}$, Farzana Chowdhary ${ }^{1}$ \\ ${ }^{1}$ Institute of Pharmaceutical Sciences, University of Veterinary and Animal Sciences, Lahore, Pakistan \\ Head of Institute: Dr. Farzana Chowdhary \\ 2Department of Pharmacy, Faculty of Pharmacy and Alternative Medicine, Islamia University of Bahawalpur, Bahawalpur, Pakistan \\ Head of Department: Prof. Dr. Naveed Akhtar
}

Postep Derm Alergol 2014; XXXI, 2: 71-76

DOI: $10.5114 / p d i a .2014 .40945$

\begin{abstract}
Introduction: Solar ultraviolet exposure is the main cause of skin damage by initiation of reactive oxygen species (ROS) leading to skin collagen imperfection and eventually skin roughness. This can be reduced by proper revitalization of skin enhancing younger and healthier appearance.

Aim: To evaluate the skin facial revitalization effect of a cream formulation containing the Moringa oleifera leaf extract on humans.

Material and methods: Active cream containing 3\% of the concentrated extract of moringa leaves was developed by entrapping in the inner aqueous phase of cream. Base contained no extract. Skin revitalizing parameters, i.e. surface, volume, texture parameters and surface evaluation of the living skin (SELS) were assessed comparatively after application of the base and active cream on human face using Visioscan ${ }^{\circledast}$ VC 98 for a period of 3 months.

Results: Surface values were increased by the base and decreased by the active cream. Effects produced for the base and active cream were significant and insignificant, respectively, as observed in the case of surface. Unlike the base, the active cream showed significant effects on skin volume, texture parameters (energy, variance and contrast) and SELS, SEr (skin roughness), SEsc (skin scaliness), SEsm (skin smoothness), and SEw (skin wrinkles) parameters.

Conclusions: The results suggested that moringa cream enhances skin revitalization effect and supports anti-aging skin effects.
\end{abstract}

Key words: Moringa oleifera, cream, facial revitalization.

\section{Introduction}

In the recent years the development of methods of skin revitalization, i.e. of its restoration after damage of any nature (including aging) has increased [1]. Solar ultraviolet exposure is the main cause of skin damage by initiation of reactive oxygen species (ROS) [2, 3]. These can interfere with proteins; fatty acids and saccharides resulted in oxidative damage [4] leading to skin collagen imperfection and eventually skin roughness [5]. Aging is associated with morphological changes [1] and is characterized by loss of elasticity, increased wrinkling, irregular pigmentation, dryness and roughness [6, 7]. Recently, topical formulations have gained considerable attention and interest as a vehicle for the drug delivery to the human skin [8]. One approach in skin protection against reducing skin aging is the use of plant phenolics and antioxidants topically [5].

Moringa oleifera (Moringaceae) is a pan-tropical species [9]. Moringa leaves are reported to be rich in phenolics and antioxidants like vitamin C, B and A [10]. Leaves are used as purgative, applied as the poultice to sores, rubbed on the temples for headaches, used for piles, fevers, sore throat, bronchitis, eye and ear infections, anti-inflammatory, scurvy and catarrh. It protects the human skin from environmental influences and combats premature skin aging [11].

\section{Aim}

The aim of the study was to evaluate the skin facial revitalization effect of a cream formulation containing the Moringa oleifera leaf extract on humans.

\section{Material and methods \\ Plant material}

Moringa oleifera leaves were gathered in July 2010 in Dera Ghazi Khan, Pakistan and air dried at room temperature for a period of 4 weeks.

Address for correspondence: Atif Ali PhD, Institute of Pharmaceutical Sciences, University of Veterinary and Animal Sciences, Lahore, Pakistan, phone: +923346081922, e-mail: ajmaline2000@gmail.com Received: 31.07.2013, accepted: 3.11.2013. 


\section{Identification of the plant}

Identification of the plant ( $M$. oleifera) was made at the Cholistan Institute of Desert Studies (CIDS), the Islamia University of Bahawalpur, Pakistan. The specimen (voucher Number: MO-LE-09-10-31) was deposited in the Herbarium of the Islamia University Bahawalpur.

\section{Materials}

Abil EM 90 was procured from Franken Chemicals, Germany, paraffin oil from Merck, Germany, methanol and phosphoric acid from BDH, England. Deionized water was obtained in the Pharmaceutical Labs of Department of Pharmacy, the Islamia University of Bahawalpur, Bahawalpur-Pakistan.

\section{Preparation of the formulation}

An active cream was prepared by an anionic hydrophilic colloid (14\% of Paraffin oil), 2\% of Abil EM 90, 3\% of $M$. oleifera leaf extract, $0.2 \%$ of phosphoric acid, $1 \%$ of fragrance and rest of deionized water. Heated oily phase and aqueous phase were mixed using homogenizer (Euro-Star, IKAD 230, Germany) 5000 rpm for $15 \mathrm{~min}, 1000 \mathrm{rpm}$ for $10 \mathrm{~min}$ and 500 rpm, by addition of phosphoric acid, extract and fragrance till cooled. The same method was adopted to prepare the base without the extract.

\section{Subjects}

Eleven subjects were selected aged between 20 and 35 years [12]. All subjects were healthy males with no known dermatological diseases or allergy to substance in formulations. The Declaration of Helsinki was followed in this single blind and controlled trial. Informed consent was signed before the start of this study by all volunteers. The exclusion criteria were as follows: presence of any dermatitis and/or other skin or allergic diseases, smokers and previous treatment of cheeks' skin with cosmetic formulations such as sunscreens, moisturizers or anti-aging cosmetics. The volunteers were asked not to apply any topical products on cheeks $24 \mathrm{~h}$. before the beginning and throughout the test period. Additionally, solar exposure and use of occlusive clothes on the test area were forbidden.

\section{Instrumental assessment}

Visioscan ${ }^{\oplus}$ VC 98 is a special UVA-light video camera with high resolution to study the skin parameters directly. The images show the structure of the skin and the level of dryness very impressively. The camera can be connected to the computer by the digitalization unit Video Digitizer VD 300 via FireWire port. All measurements were made in a draught-free room, with controlled temperature $\left(18.0-20.6^{\circ} \mathrm{C}\right)$ and relative humidity (50-65\%).

\section{Study protocol}

In vivo investigations have been carried out during the winter months (October 2011 to January 2012). All instrumental measurements were done by the author according to manufacturer's instructions. Two weeks before the study beginning and during the treatment period, the volunteers were permitted only to use normal cleansing products. Each volunteer was then handed two creams, the active cream containing the plant extract and the base without the extract. The volunteers were well informed about the correct use of the creams. Measurements of skin parameters, which include surface, volume, texture parameters and surface evaluation of living skin (SELS), were done every month up to the end of the study period of 3 months. Approximately, $500 \mathrm{mg}$ of both active cream and base were to be applied to the cheeks twice daily (mornings, 7:00-9:00; evenings, 19:00-21:00) over a 12-week period at home by the volunteers. The area around the eyes was omitted. Before all measurements, volunteers remained in the experimental room for at least $15 \mathrm{~min}$ in order to tolerate skin adjustment to room temperature.

\section{Ethical standards}

The approval of this study (Ref. No. 3715/Acad) was obtained from the Advanced Study and Research Board (ASARB), the Islamia University of Bahawalpur, Bahawalpur and the Institutional Ethical Committee, Faculty of Pharmacy and Alternative Medicine, the Islamia University of Bahawalpur, Bahawalpur-Pakistan.

\section{Mathematical and statistical analysis}

The skin parameter values (surface, volume, texture parameters and surface evaluation of living skin) of the right and left cheek of the volunteers were calculated at $0 \mathrm{~h}, 1^{\text {st }}, 2^{\text {nd }}$ and $3^{\text {rd }}$ month (Figure 1). SPSS 17.0 was used for data analysis on the computer by using the two-way ANOVA for variation at different time intervals and the paired sample $t$-test for the variation between the two formulations. The level of significance was set at $p<0.05$.

\section{Results and discussion}

The values for the surface, volume, texture parameters and SELS were taken by Visioscan ${ }^{\circledR}$ VC 98 before application of the creams ( $0 \mathrm{~h}$ readings), then after $1^{\text {st }}, 2^{\text {nd }}$ and $3^{\text {rd }}$ month of the study period.

\section{Surface and volume}

Surface values (smoothness of the skin) is directly associated with the micro-furrows and furrows. Less micro-furrows and furrows are indicative of smoothness of skin and ultimately reduction in wrinkles [13]. In our results, surface values are constantly decreased, which is produced by the active cream and are increased by the 

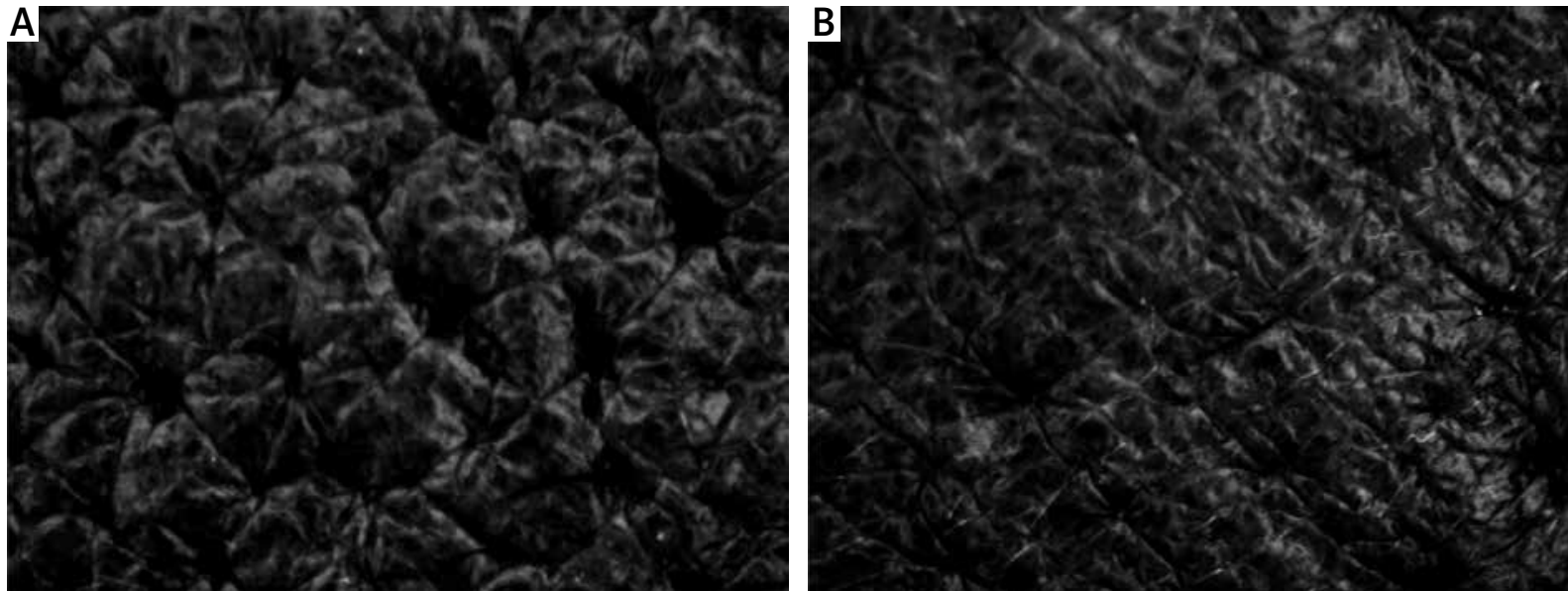

Figure 1. The right cheek of a human volunteer (A) before and (B) after application of active cream for 3 months

base after application when assessed by Visioscan ${ }^{\oplus}$ VC 98 (Figure 2). The base produced statistically significant effects at all reading intervals on the surface parameters, and active cream produced insignificant effects when two-way ANOVA was applied. When paired t-test was applied, it was observed that the active cream produced significant results in volunteers except the $2^{\text {nd }}$ month. A positive surface reduction is associated with reduction in depth of micro-wrinkles and reduction in skin roughness [14]. However, in our results reduction was achieved but not significant surface changes by moringa cream.

Volume measures the virtual amount of liquid $\left(\mathrm{mm}^{3}\right)$ required to fill the depths in image. In young and smooth surface of the skin less virtual liquid is required to fill the depths. The active cream produced significant effects when two-way ANOVA was performed. Descending val-

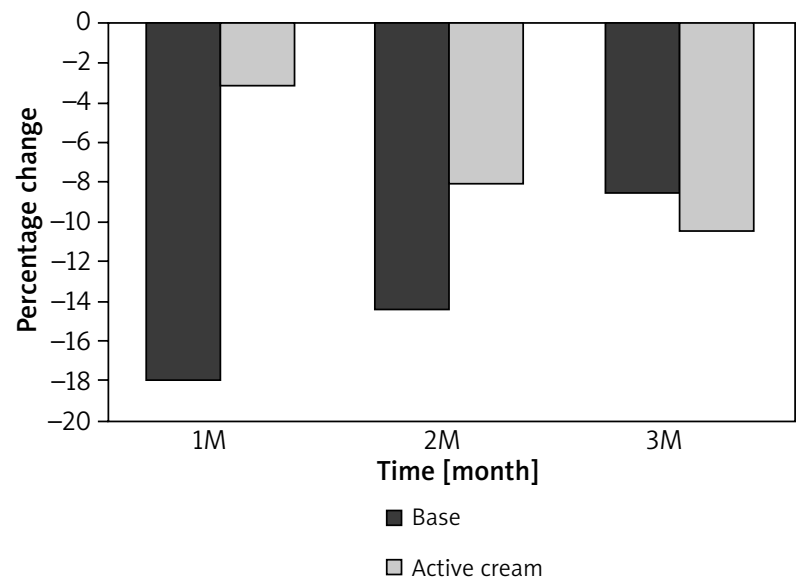

$1 M$ - application of the base and active cream after 1 month, $2 M$ - application of the base and active cream after 2 months, $3 M$ - application of the base and active cream after 3 months

Figure 2. Change (\%) in the surface values of volunteers after the application of the base and active cream ues were observed for the active cream. The active cream also showed significant effects when paired sample t-test was applied for variation between the two creams. This supports our findings of decreased volume values by the active cream until the $12^{\text {th }}$ week when applied resulting in skin smoothness and less rough skin as less amount of virtual liquid is required as shown in Figure 3.

\section{Texture parameters}

The parameters (energy, contrast and variance) analyze differences in colors of neighboring pixels [13]. Effects of texture parameters produced by the active cream and base on volunteers are shown in Figure 4. Energy is increased with an increased hydration level of the skin and more homogeneity of an image [15]. In this study, an

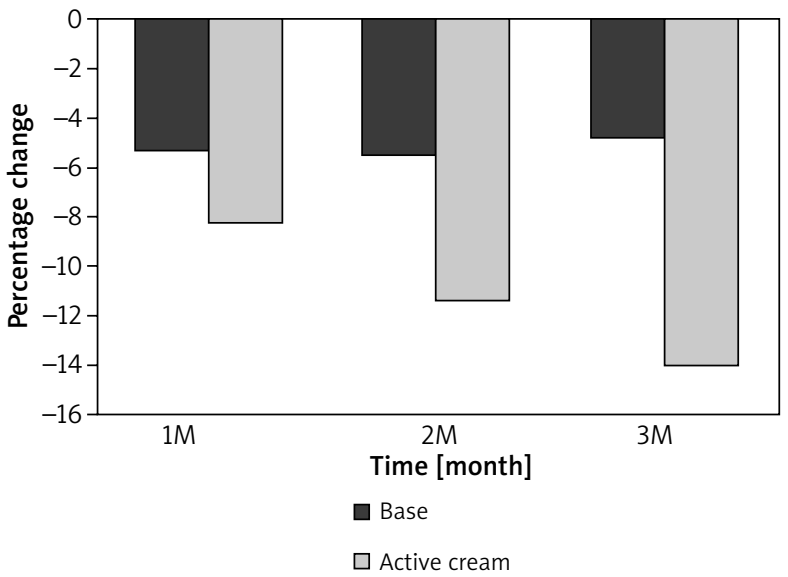

$1 M$ - application of the base and active cream after 1 month, $2 M$ - application of the base and active cream after 2 months, $3 M$-application of the base and active cream after 3 months

Figure 3. Change (\%) in the volume values of volunteers after the application of the base and active cream 


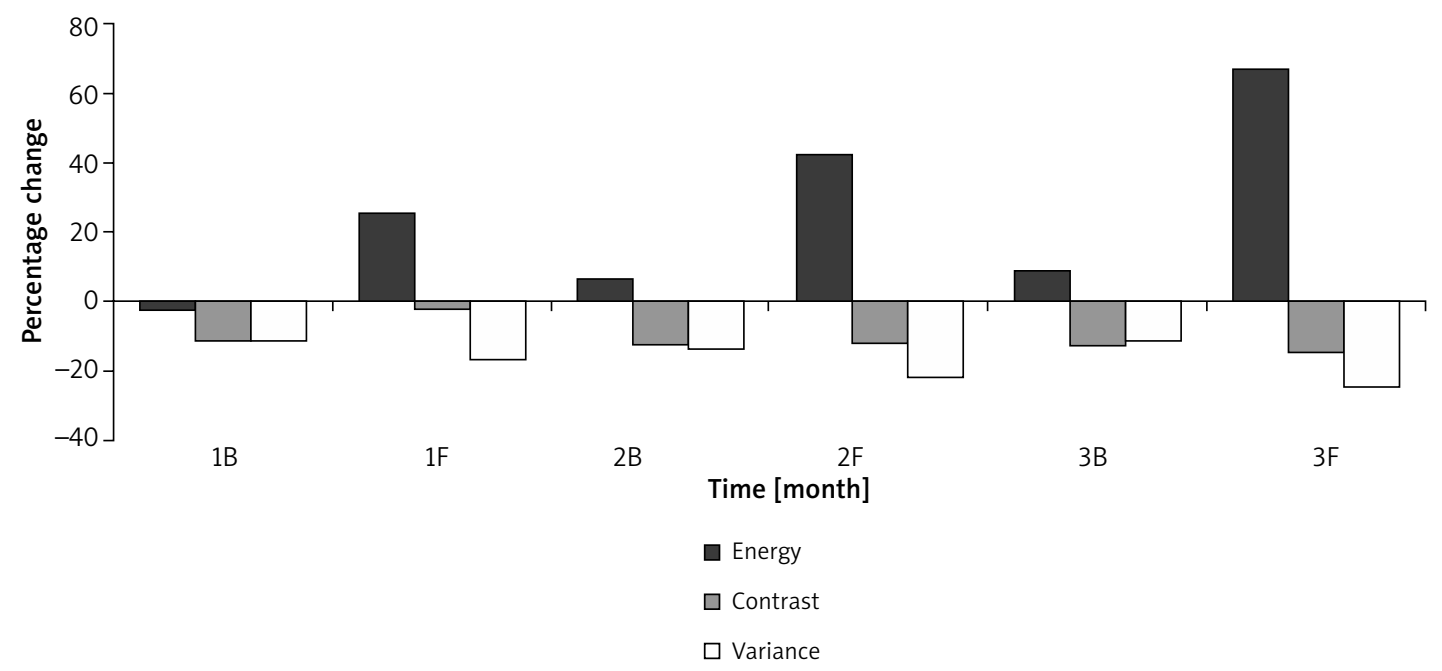

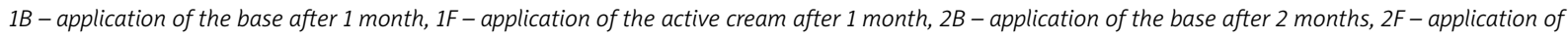
the active cream after 2 months, $3 B$-application of the base after 3 months, $3 F$-application of the active cream after 3 months

Figure 4. Change (\%) in skin texture parameter (energy, contrast and variance) of volunteers with time after following application of base and active cream

increase in the energy values for the active cream was statistically significant at all reading intervals, but the base produced insignificant effects. The active cream also showed significant effects when paired sample $t$-test was applied for variation between the two creams except the $3^{\text {rd }}$ month. Moisturizing and anti-aging treatment provides energy when applied so this study needs to assess moisturization effects of the active cream evaluated by a corneometer to support that either active cream may provide hydration to the Stratum corneum. Our findings of an increase in energy parameters indicated a general overview over the state of skin as moisturizer. A significant result of energy obtained by moringa cream was indicative of more elastic and hydrated skin in human volunteers.

Variance is the average of a local variance over a number of pixels. The actual value of the pixel is compared to the average. High variance indicates high roughness of skin surface [16]. In this study, a decrease in the variance values for the active cream was statistically significant at all reading intervals but the base produced insignificant effects. The active cream also showed significant effects when paired sample t-test was applied for variation between the two creams. Our findings disclosed a reduction in variance, which supports less roughness of the skin surface.

Contrast indicates the difference between gray levels of the two neighboring pixels. The higher the contrast, the higher the different values of two neighbors. A good skin condition will show low contrast values [16]. In this study, a decrease in the contrast values for the active cream was statistically significant at all reading intervals but the base produced insignificant effects. The active cream showed significant effects when paired sample t-test was applied for variation between the two creams except the $1^{\text {st }}$ and $2^{\text {nd }}$ month. Reduction in contrast showed a better condition and smoothness of the skin resulting in the reduction in roughness.

Retaining a proper water gradient in the skin is essential for maintenance of mechanical properties of collagen as well as elastic fibers. Collagen and elastin retain their three-dimensional structure in the hydrated form only - water deficiency leads to altered physical qualities of the proteins [16]. Enhanced elasticity of the skin on the cheeks after the use of the moringa leaf extract-enriched cream may result from proper skin hydration and at the same time from normal activity of fibroblasts responsible for collagen synthesis. The presence of the moringa leaf extract contributed to an enhanced division of fibroblasts, increased metabolism, including turnover of collagen and elastin. Mechanical properties of the skin (elasticity) depend on the structure and density of collagen and elastic fibers. Normally functioning skin does not lose the ability to contract and does not get slack, while these factors contribute to the development of wrinkles.

\section{Surface evaluation of living skin}

The percentage changes of different SELS parameters SEr, SESC, SESm and SEW measured by Visioscan ${ }^{\circledR}$ VC 98 before application of creams and at the $1^{\text {st }}, 2^{\text {nd }}$ and $3^{\text {rd }}$ month of the study period are given in Figure 5. SEr is the roughness parameter, which calculates the proportion of dark pixels. SEsm is the index of smoothness and is cal- 


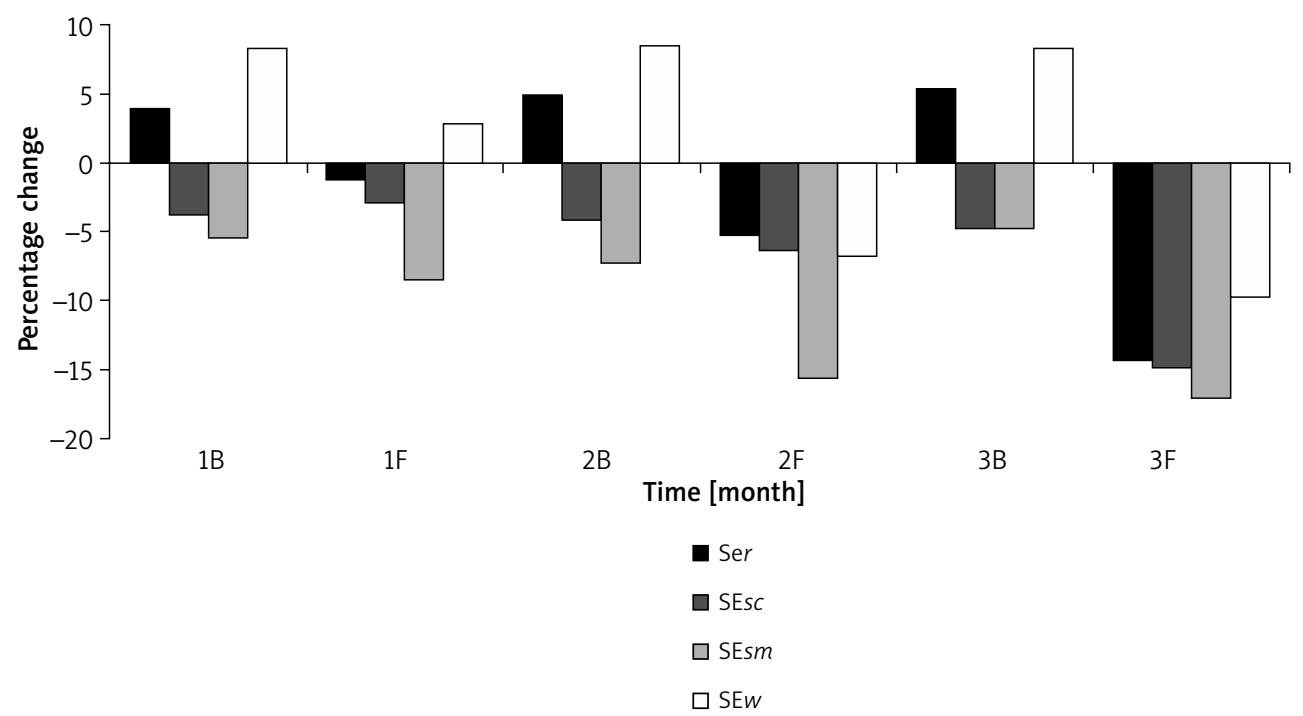

$1 B$-application of base after 1 month, $1 F$-application of the active cream after 1 month, 2B-application of the base after 2 months, $2 F-$ application of active cream after 2 months, $3 B$-application of the base after 3 months, $3 F$-application of active cream after 3 months

Figure 5. Change (\%) in SELS parameters of volunteers' skin following application of base and active cream

culated from the mean width and depth of wrinkles. SESC is the index of scaliness of skin, which shows the level of skin dryness. SEW identifies aging including wrinkles and is calculated from the proportion of horizontal and vertical wrinkles [17]. In this study, it was found that the base produced statistically insignificant effects on the roughness parameter of the skin, and the active cream produced significant effects at all reading intervals when ANOVA two way analysis was performed. When paired sample $t$-test was applied for SEr, SESC, SEsm and Sew, significant effects were observed for the active cream except the $1^{\text {st }}$ and $2^{\text {nd }}$ month in the case of Ser, $3^{\text {rd }}$ month in the case of SESC, $1^{\text {st }}$ and $3^{\text {rd }}$ month in the case SEsm and $1^{\text {st }}$ and $3^{\text {rd }}$ month in the case of SEW. A gradual decrease in the values of roughness, scaliness, smoothness and wrinkles were observed for the active cream, which indicated that the active cream had anti-aging properties. The smaller SESC value corresponds to higher skin moisture as treatment with anti-aging formulations resulted in lower values for SESC. Lower values for the SEW parameter indicated that there were fewer wrinkles present on the skin, which indicates that the active cream reduced the fine wrinkles. This was directly related to the gain of collagen.

It is assumed that the improvement in skin surface parameters can be attributed to the moringa phenolics which include kaempferol, quercetin, rutin, gallic, chlorogenic, ellagic and ferulic acid and antioxidants, which include vita$\min A, C$ and $B$. Phenolics offer the best protection against enzymes that cause the breakdown of collagen and elastin in skin, ultimately skin rejuvenation [10, 11]. $\beta$-Carotene has been revealed to have topical photo-protective outcomes in an increase in protein and collagen as well as DNA con- tent and increased epidermal thickening [18]. Vitamin B functions as a humectant and increases the water content. Humectants can attract water into the Stratum corneum to soften the skin; this is effective as a moisturizer [18]. All of these investigations collectively support our findings that the active cream supports anti-aging properties.

\section{Conclusions}

The results achieved in this investigation suggest that topical formulation of moringa extract is capable of revitalizing the skin and reducing signs of skin aging. Future studies are required to unravel the anti-aging activity and mechanism of plant constituents in the form of topical formulation.

\section{Acknowledgments}

The authors wish to thank the Higher Education Commission of Pakistan for providing financial support to the study. The authors also acknowledge moral support given by the Chairman and Dean of the Faculty of Pharmacy and Alternative Medicine, the Islamia University of Bahawalpur, Pakistan.

\section{Conflict of interest}

All authors declare no conflict of interest.

\section{References}

1. Flynn TC, Coleman WP. Topical revitalization of body skin. J Eur Acad Dermatol Venereol 2002; 14: 280-4. 
2. González S, Fernández-Lorente M, Gilaberte-Calzada Y. The latest on skin photoprotection. Clinic Dermatol 2008; 26: 614-26.

3. Sowa P, Rutkowska-Talipska J, Rutkowski K, et al. Optical radiation in modern medicine. Postep Derm Alergol 2013; 30: 246-51.

4. Svobodova A, Psotova J, Walterová D. Natural phenolics in the prevention of UV-induced skin damage. A review. Biomed Pap Med Fac Univ Palacky Olomouc Czech Repub 2003; 147: 137-45.

5. Ichihashi M, Ueda M, Budiyanto A, et al. UV-induced skin damage. Toxicology 2003; 15: 21-39.

6. Fisher GJ, Kang S, Varani J, et al. Mechanisms of photoaging and chronological skin aging. Arch Dermatol 2002; 138: 1462-70.

7. Pawlaczyk M, Lelonkiewicz M, Wieczorowski M. Age-dependent biomechanical properties of the skin. Postep Derm Alergol 2013; 30: 302-6.

8. Foldvari M. Non-invasive administration of drugs through the skin: challenges in delivery system design. Pharm Sci Technol Today 2000; 3: 417-25.

9. Iqbal S, Bhanger M. Effect of season and production location on antioxidant activity of Moringa oleifera leaves grown in Pakistan. J Food Compos Anal 2006; 19: 544-51.

10. Manguro LOA, Lemmen P. Phenolics of Moringa oleifera leaves. Nat Prod Res 2007; 21: 56-68.

11. Anwar F, Latif S, Ashraf M, Gilani AH. Moringa oleifera: a food plant with multiple medicinal uses. Phytother Res 2007; 25: 17-25.

12. Atif A, Naveed A, Muhammad SK. In vivo evaluation: the effects of a cream containing Acacia bark extract on skin melanin and erythema content. Postep Derm Alergol 2012; 29: 369-72.

13. Visioscan ${ }^{\oplus}$ VC 98 and the Software SELS (Surface Evaluation of the Living Skin) Courage and Kazaka. 2009; 1-53.

14. Béguin A. A novel micronutrient supplement in skin aging: a randomized placebo-controlled double-blind study. J Cosmetic Dermatol 2005; 4: 277-84.

15. Rasul A, Akhtar N. Formulation and in vivo evaluation for anti-aging effects of an emulsion containing basil extract using non-invasive biophysical techniques. Daru 2011; 19: 344-50.

16. Debowska R, Rogiewicz K, Iwanenko T, et al. Folic acid (Folacin) - new application of a cosmetic ingredient. Kosmetische Medizin 2005; 3: 16-22.

17. Ishii H, Kitamura T, Todo H, et al. Combined effect of sodium chondroitin sulfate and sodium hyaluronate on skin moisturization following single and repeated application. Asian J Pharm Sci 2008; 3: 94-101.

18. Lupo MP. Antioxidants and vitamins in cosmetics. Clin Dermatol 2001; 19: 467-73. 